\title{
Editorial
}

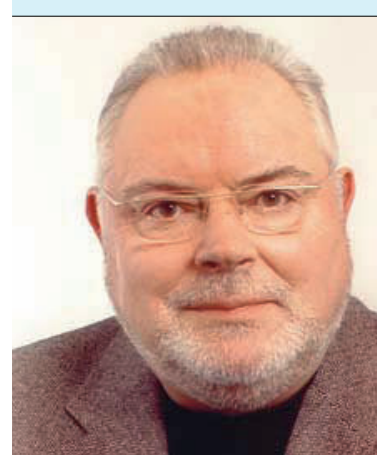

„Wer den Lärmpegel ständig hoch hält, braucht sich

nicht zu wundern, wenn seine Botschaften irgendwann

auf taube Ohren stoßen."

Dr. med. Dieter Leithäuser

Chefredakteur und niedergelassener HNO-Arzt, Warburg

\section{Von lauten und von leisen Tönen}

$\mathrm{D}$ ie Fußball-WM ist vorbei, verstummt sind die Vuvuzelas. Immerhin wurden unsere Innenohren mit $127 \mathrm{~dB}$ getroffen. Phonak hat reagiert und sogleich den Vergleich mit anderen, ähnlichen Spitzenwerten geliefert: die Kettensäge mit $120 \mathrm{~dB}$, ein Schlagzeug mit $122 \mathrm{~dB}$ und die Schiedsrichterpfeife mit 121,8 dB. Ohrstöpsel Marke Vuvuzela-Ex oder das simple deutsche Oropax waren ausverkauft (immerhin konnten sie laut Werbung auch gegen keifende Ehefrauen eingesetzt werden). Wir wollen hoffen, dass die durch die röte innenohrgeschädigten Menschen wenigstens in unseren HNO-Praxen gelandet sind und brav ihre 10 Euro Praxisgebühr entrichtet haben.

Aber wie würde sich der Vorschlag der Kassenärztlichen Bundesvereinigung auswirken, 5 Euro bei jedem Praxisbesuch zu kassieren? Damit sollen diejenigen „Patienten“" getroffen werden, die zum Arzt gehen, obwohl sie nicht krank sind. Möglicherweise ist so mancher ja wirklich nicht krank, sondern will nur kostenlos die Zeitschriften im Wartezimmer lesen. Und wenn er schon 5 Euro zahlt, soll es dann doch bitte schön mindestens die aktuelle Bunte oder der Stern sein oder der neue Focus mit den „100 besten HNO Ärzten“ und „Deutschlands billigsten Bestattern“. Eine eher kärgliche Zeitschriften-Auswahl und ein zerfledderter Stapel mit der Alpenvereinszeitschrift von 1970 befriedigt da nicht. Am besten stellen Sie auch noch ein Aquarium in Ihr Wartezimmer, da „werden Sie auch visuell schnell geholfen“. Mit der geplanten Praxisgebührenregelung wird der Arztbesuch quasi in eine neue Erlebnisqualität gebeamt und zur Blutabnahme bei Jugendlichen erscheint eine als Vampir verkleidete Sprechstundenhilfe.
Ganz leise Töne haben Forscher der Universität London entdeckt: Schon sieben Monate alte Babys wissen genau, ob ihre Mama verärgert ist oder nicht. Sie können das am Klang der Stimme erkennen. Kleinkinder reagieren schon in diesem Alter auf Emotionen, die in der Stimme mitschwingen. Die Hirnaktivität der Babys, mit der sie auf Stimmen reagieren, ähnelt bereits weitgehend der von Erwachsenen. Die menschliche Stimme übermittelt nicht einfach nur die gesprochenen Worte, sondern eben auch Gefühlslagen. Das geschieht vor allem über die Satzmelodie. Ab wann wir diese Zwischentöne heraushören, war bisher nicht erforscht. Nun zeigte sich, dass eine genaue Stimmenerkennung sich bereits im Alter zwischen vier und sieben Monaten ausbildet. Mit dieser Erkenntnis, so die Forscher, könne man künftig Entwicklungsstörungen wie etwa Autismus besser diagnostizieren.

Aber zurück zur Vuvuzela: Sogar bei evangelischen Kirchengemeinden kam sie gut an: Deren Posaunenchöre haben dem trompetenförmigen Blasinstrument ganz neue Töne entlockt und selbstkomponierte Fußballfanfaren ins Internet gestellt. Was man nun besser findet, laut oder leise, bleibt jedem selbst überlassen.

\section{Ihr Dieter Leithäuser}

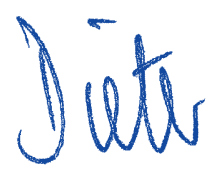

